\title{
Uncloned expanded CAG/CTG repeat sequences in autosomal dominant cerebellar ataxia (ADCA) detected by the repeat expansion detection (RED) method
}

Miguel Angel Pujana, Víctor Volpini, Mónica Gratacós, Jordi Corral, Isabel Banchs, Aurora Sánchez, David Genís, Carlos Cervera, Xavier Estivill

\begin{abstract}
In some neurodegenerative diseases, genetic anticipation correlates with expansions of the CAG/CTG repeat sequence above the normal range through the generations of a pedigree. Among these neurodegenerative diseases are late onset autosomal dominant cerebellar ataxias (ADCA). ADCA are genetically heterogeneous disorders with different cloned genes for spinocerebellar ataxia type 1 (SCA1), type 2 (SCA2), type 3 or Machado-Joseph disease (SCA3/MJD), and type 6 (SCA6). Another related dominant ataxia, dentatorubral-pallidoluysian atrophy (DRPLA), also shows CAG/CTG repeat expansions. Genetic anticipation has been reported for all of them except for the recently cloned SCA6 gene. Other, as yet undetected SCA genes may show the same features. We have used the repeat expansion detection (RED) method to detect repeat expansions directly in DNA samples from ADCA patients not resulting from known genes. Our sample consists of 19 affected index cases, corresponding to $52.8 \%$ of our ADCA families without CAG/CTG repeat expansions in the SCA1, SCA2, SCA3/MJD, SCA6, or DRPLA genes. Eighty-nine percent of the index cases had expansions of a CAG/CTG sequence greater than 40 repeats by RED, while these were observed in only $26.9 \%$ of 78 healthy subjects from the general population $(p<0.0001)$. The distribution of RED fragments in controls and ADCA patients also shows significant differences with the Mann-Whitney $U$ test $(U=376.5, p=0.0007)$. Moreover, there was a significant inverse correlation between the size of expansion and the age of onset $(r=-0.54, p=0.018)$. These results show CAG/CTG repeat expansions of over 40 repeats in our sample of ADCA families not resulting from known SCA genes. (F Med Genet 1998;35:99-102)
\end{abstract}

Keywords: dominant ataxia; CAG repeats; repeat expansion detection

Several neuropathological diseases show increasing severity and a decrease in the age of onset through the generations of a pedigree, a phenomenon known as anticipation. ${ }^{1}$ Among other neuropathological diseases, such as Huntington's disease ${ }^{2}$ or $\mathrm{X}$ linked recessive bulbospinal neuronopathy, ${ }^{3}$ some familial late onset cerebellar ataxia pedigrees clearly show genetic anticipation. These severe neurodegenerative diseases have a monogenic autosomal mode of inheritance (with the exception of bulbospinal neuronopathy) with age dependent penetrance and variable expressivity. Neuropathological studies usually show atrophy and loss of neuronal cells in the cerebellar cortex, encephalic trunk, basal ganglia, and spinal cord. Besides ataxia, dementia, pyramidal and extrapyramidal signs, and oculomotor and spinal cord dysfunction have been described (SCA, spinocerebellar ataxia; OPCA, olivopontocerebellar ataxia; ADCA, autosomal dominant cerebellar ataxia). ${ }^{4}$

At the molecular level, anticipation correlates with expansions of certain trinucleotide repeats above the normal range. Five genes for late onset autosomal dominant ataxia diseases caused by CAG/CTG repeat expansions have been cloned: the SCA1 gene, ${ }^{5}$ the SCA2 gene, ${ }^{6-8}$ the SCA3/MJD gene, ${ }^{9}$ the SCA6 gene, ${ }^{10}$ and one gene for a related dominant ataxia, dentatorubral-pallidoluysian atrophy (DRPLA). ${ }^{11}{ }^{12}$ An inverse correlation between the repeat length and the age of onset, which results in genetic anticipation, has been reported for these genes, ${ }^{6-8}{ }^{13-15}$ except for the small polyglutamine expansion in SCA6. The CAG/CTG repeat sequence encodes a polyglutamine tract that is mutated when the allele has expansions beyond the normal range, typically over 40 repeats, except for SCA 6 which does not exceed 30 repeats. Other late onset autosomal dominant cerebellar ataxia loci have been mapped to chromosomes 3p (SCA7), ${ }^{16}{ }^{17}$ $11 \mathrm{q}$ (SCA5), ${ }^{18}$ and 16q (SCA4). ${ }^{19}$ These loci could harbour genes with the same features as described above, as has been shown for SCA $7,{ }^{20}{ }^{21}$ the locus that represents ADCA type II (ADCA with retinopathy).

The repeat expansion detection (RED) method $^{22}$ allows the detection of the presence of trinucleotide repeat expansions in a DNA sample by cycles of annealing and ligation of a repeat specific oligonucleotide. It had previously been used to determine the approximate length of the CTG/GAC, CGG/GCC, and CAG/CTG repeat expansions in myotonic dystrophy, fragile $\mathrm{X}$ syndrome, and Huntington's disease (HD).$^{22}{ }^{23}$ It has also been applied to the detection of possible CAG/CTG repeat 
Table 1 Repeat expansion detection (RED) analysis in 19 patients with autosomal dominant cerebellar ataxia not resulting from SCA1, SCA2, SCA3, SCA6, or DRPLA expansions and in healthy subjects

\begin{tabular}{lcc}
\hline CAG/CTG repeat & Ataxics & Controls \\
\hline 24 & 1 & 8 \\
32 & 0 & 18 \\
40 & 1 & 31 \\
48 & 10 & 3 \\
56 & 3 & 5 \\
64 & 1 & 7 \\
72 & 0 & 1 \\
80 & 1 & 0 \\
88 & 2 & 0 \\
$>120$ & 0 & 5 \\
& 19 & 78
\end{tabular}

Mann-Whitney U test.

Controls=median $40(\mathrm{Q} 25=32 ; \mathrm{Q} 75=48)$.

Ataxics $=$ median $48(\mathrm{Q} 25=48 ; \mathrm{Q} 75=56)$.

$\mathrm{U}=376.5 ; \mathrm{p}=0.0007$.

Table 2 Ages of onset and repeat expansion detection (RED) results for 19 patients with autosomal dominant cerebellar ataxia ( $A D C A)$

\begin{tabular}{lll}
\hline No of patients & Age of onset & RED result \\
\hline 2 & 10 & 56,80 \\
1 & 22 & 88 \\
3 & 25 & $48,56,88$ \\
1 & 30 & 40 \\
1 & 33 & 48 \\
2 & 40 & 48 \\
2 & 41 & 48 \\
1 & 46 & 64 \\
2 & 50 & 24,56 \\
1 & 52 & 48 \\
2 & 55 & 48 \\
1 & 60 & 48 \\
\hline
\end{tabular}

$\mathrm{r}=-0.54, \mathrm{p}=0.018$.

expansions in Parkinson's disease, ${ }^{24}$ schizophrenia, ${ }^{25-28}$ and bipolar depressive disorder. ${ }^{26}$ We have previously applied an improved RED method to SCA1, SCA3/MJD, and $H D$ patients where the size of the repeat expansion was already known and have shown an excellent estimation of the CAG/CTG expansion in each sample. ${ }^{29}$ We present here the results of the RED method applied to ascertain whether as yet uncloned genes for ADCA could have CAG/CTG repeat expansions.

\section{Materials and methods SUBJECTS}

We have studied 19 index cases from 19 Spanish families with documented ADCA. These represent $52.8 \%$ of our ADCA families. None of these families has clinical features of ADCA type II (with retinopathy). The diagnosis was based on the classical clinical criteria of this neurodegenerative disorder after neurological examination, computerised tomography (CT), or magnetic resonance imaging (MRI). The 19 index cases and the other affected members of each family were initially analysed for expansions in known disease genes, SCA1, SCA2, SCA3/MJD, SCA6, and DRPLA, showing CAG/CTG repeats in the normal range. None of these 19 ADCA families was large enough for linkage studies with markers for other described ADCA loci. We have ascertained the age of onset in all of the 19 index cases. Seventy-eight healthy, unrelated subjects from the general population were used as control samples. All the subjects of the study were from Spain and were of European origin.

RED METHOD

Genomic DNA was isolated from peripheral blood lymphocytes using proteinase K/SDS digestion and a salt extraction. We used an 8-mer oligonucleotide that improves the previous size resolution achieved with 10- or 17-mer oligonucleotides. This oligonucleotide size allowed us to detect small differences in sizes that eventually could be correlated with anticipation. The reaction was performed using $7 \mu \mathrm{g}$ of each DNA sample from the index cases, with previously reported reaction conditions. ${ }^{22}{ }^{30}$ In order to test the power of detection of the method, we previously analysed known CAG/ CTG repeat expansions in $\mathrm{HD}, \mathrm{MJD}$, and SCA1 patients, obtaining in all cases ligation bands that corresponded to the expected size of the sequenced alleles. ${ }^{29}$

\section{ASSESSMENT OF CAG/CTG LIGATION PRODUCTS}

The RED method consists of cycles of reaction in which oligonucleotides bind to the repeat stretch in the template DNA, while the $3^{\prime}$ and 5' from adjacent oligonucleotides are covalently bound by a thermostable ligase. The reaction is performed until all sites in the template DNA are occupied by oligonucleotides. Then the ligation products are electrophoresed and the bands visualised by hybridisation with a labelled complementary oligonucleotide. The largest ligation product obtained per sample, which is a multiple of 8 in our case, is taken as the size of the repeat expansion. In autosomal dominant cerebellar ataxias, the number of repeats in affected subjects was observed to be between 35-40 and 90 repeats except for SCA6, which expands between 21 and 27 repeats. Thus, we have assigned RED disease alleles when they are $\geqslant 48$ CAG/CTG repeats.

\section{STATISTICAL ANALYSES}

The Mann-Whitney U-Wilcoxon test was performed with SPSS 6.1 (SPSS Inc, Chicago) on two groups, controls and ADCA index cases.

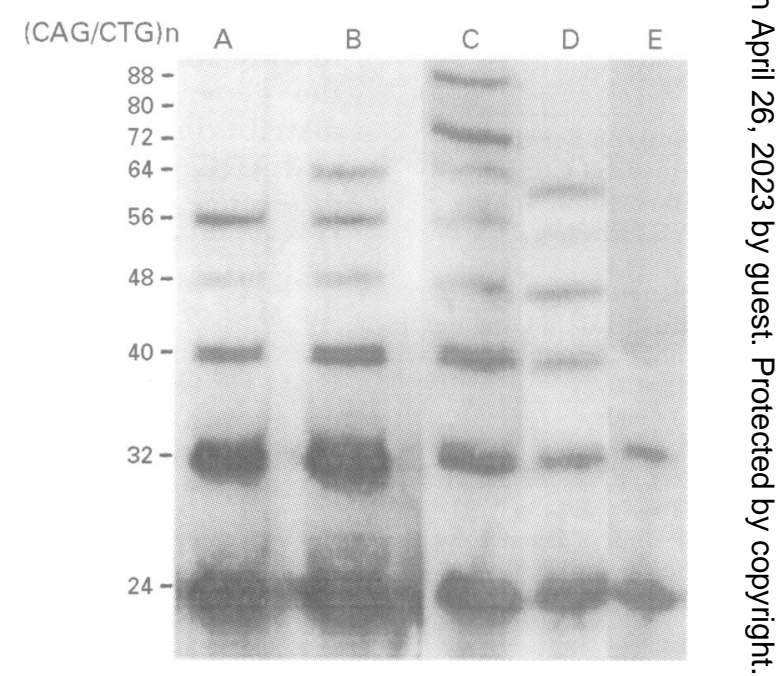

Figure 1 CAG/CTG repeat analysis by RED in $A D C A$ and control samples: $(A) 56$ repeats, $(B)$ and $(D) 64$ repeats, $(C) 88$ repeats, and $(E) 32$ repeats. The numbers on the left are the CAG/CTG repeat sizes obtained by $R E D$ using an 8-mer oligonucleotide. 
The comparison of results obtained in ADCA index cases and healthy subjects from the general population were performed using chisquared analysis in a two by two contingency table with classifications of "disease allele" or "not disease allele", and "affected" or "unaffected". Additionally, a regression analysis was conducted to measure the degree of association between age of onset and CAG/CTG repeat expansion. Statistical differences were considered at the level of $\mathrm{p}<0.05$.

\section{Results}

None of the affected subjects from the 19 ataxia families showed CAG/CTG repeat expansions in the SCA1, SCA2, SCA3/MJD, SCA6, or DRPLA genes. Owing to the small size of the pedigrees and lack of informativeness, we could not exclude linkage at the SCA4, SCA5, or SCA7 loci in these families. One index case (1/19) showed a ligation product of fewer than 40 repeats (24 repeats). Another index case (1/19) had a ligation molecule of 40 repeats. The remaining index cases $(17 / 19,89.5 \%)$ had expanded CAG/ CTG ligation molecules ranging between 48 and 88 repeats (fig 1 ). The 78 control samples from the general population had ligation molecules greater than 40 repeats in $26.9 \%$ of cases $(21 / 78)(p<0.0001)$. The distribution of the RED products in the two populations (controls and ADCA index cases) was significantly different when using the Mann-Whitney $U$ test $(U=376.5, p=0.0007$ ) (table 1$)$. We have found a significant inverse correlation between the maximum size of the ligated CAG/CTG molecules and the respective age of onset of ataxia in the 19 index cases (table 2) $(r=-0.54$; $\mathrm{p}=0.018$ ).

\section{Discussion}

The clinical signs, age of onset, and duration of the disease vary substantially between and within ADCA families, making clinical classification extremely difficult. Heterogeneity of the ADCA genes partially explains this extensive clinical variability. Anticipation is probably the common clinical feature present in most ADCA pedigrees and it has been reported in pedigrees of dominant ataxia at loci SCA1, SCA2, SCA3/MJD, and DRPLA, ${ }^{6-8}$ 13-15 $^{15}$ and it is also present in pedigrees of ataxia that correspond to uncloned but mapped loci SCA $5,{ }^{18}$ SCA $7,{ }^{16}{ }^{17}$ and probably in SCA $4 .{ }^{19}$ In SCA7, Trottier et al, ${ }^{20}$ with a monoclonal antibody that selectively recognises polyglutamine expanded tracts, and Lindblad et al, ${ }^{21}$ using the RED method, have shown that an expanded CAG/CTG repeat sequence should exist.

We have shown, using the RED method, that the CAG/CTG repeat expansion between 48 and 88 units is a common mutation in a sample of ADCA families that do not have expansions in already cloned genes. The 48 repeat size is the most commonly found and accounts for $59 \%$ of expanded alleles detected in the ADCA index cases. The expansions accounted for $89 \%$ of the non-related index cases, in contrast to $26.9 \%$ detected in healthy subjects from the general population. Interestingly, the sizes of the RED expansions show an inverse correlation with the ages of onset, clearly indicating their pathogenic involvement as a disease causing gene. The inverse correlation observed is not as strong as in previously reported genes with CAG/CTG repeat expansions. This is probably because of the size estimation of the repeat expansion by the RED method, the genetic heterogeneity of ADCA, and the wide distribution of repeat expansions observed, suggesting that two or more disease causing loci are involved in our sample of ADCA index cases. One of our index cases did not show a CAG/CTG repeat sequence of 40 or larger. This could be because of a size distribution of expanded alleles below 40 repeats as in SCA6, or a less accessible locus by the RED method, or a type of mutation different from CAG/ CTG repeat expansion. Previous studies by Stevanin et $a l,{ }^{31}$ screening a series of ADCA patients without known expansions, using an antibody that specifically recognises large polyglutamine tracts, only detected expansions in SCA 2 and SCA7 patients. This low level of detection could be because not all of the polyglutamine expansions are expressed at levels significant enough to be detected in their lymphoblastoid system. ${ }^{31}$

Another remarkable outcome of the distribution of expanded sequences in patients versus controls from the general population was that in controls there were no expanded sequences between 80 and 88 repeats. However, these sizes were found in three affected subjects with an early age of onset. In contrast, some of the control samples $(5 / 78,6.4 \%)$ had expansions larger than 88 repeats ( $>>120$ repeats). From the data obtained so far in the genes with known CAG/CTG repeats, coding CAG/CTG repeat expansions are in the range of between 40 and 90 repeats, so it is likely that larger sizes, such as those detected in the five controls, fall in non-coding regions.

The results presented here suggest the need for further efforts to develop and apply techniques to specify cloned CAG/CTG repeat expanded sequences, such us the DIRECT method $^{8}$ or the monoclonal antibody screening method, ${ }^{6}$ which have led to the cloning of the SCA2 gene, or the screening of cDNA libraries or databases. Meanwhile, our results have shown the usefulness of the RED method applied to ADCA pedigrees, where genetic anticipation is expected. Other studies using RED should be conducted to ascertain the presence of CAG/CTG repeat expansions in sporadic cases of cerebellar ataxia.

We are very grateful to all of the families for their collaboration. This work has been supported by grants from the "Fondo de Investigaciones Sanitarias de la Seguridad Social" (95/0020 00), the European Union CT96-1364, and the "Servei Catala de la Salut". We thank Lourdes Martorell, Helena Kruyer, and
Anna Ruiz for advice.

1 Richards RI, Sutherland GR. Dynamic mutations: a new class of mutations causing human disease. Cell 1992;70:709-12.

2 Huntington's Disease Collaborative Research Group. A novel gene containing a trinucleotide repeat that is expanded and unstable on Huntington's disease chromosomes. Cell 1993;72:971-83.

3 Doyu M, Sobue G, Mukai E, et al. Severity of X-linked recessive bulbospinal neuronopathy correlates with size of 
the tandem CAG/CTG repeat in androgen receptor gene. Ann Neurol 1992;32:707-10.

4 Harding AE. Clinical features and classification of inherited ataxias. Adv Neurol 1993;61:1-13.

5 Banfi S, Servadio A, Chung M, et al. Identification and characterization of the gene causing type 1 spinocerebellar ataxia. Nat Genet 1994;7:513-20.

6 Imbert G, Saudou F, Yvert G, et al (group 1); Cancel G Abbas N, Dürr A, et al (group 2). Cloning of the gene for spinocerebellar ataxia 2 reveals a locus with high sensitivity to expanded CAG/CTG/glutamine repeats. Nat Genet 1996;13:285-91.

7 Pulst SM, Nechiporuk A, Nechiporuk T, et al. Moderate expansion of a normally biallelic trinucleotide repeat in spinocerebellar ataxia type 2 . Nat Genet 1996;13:269-76.

8 Sanpei K, Takano H, Igarashi S, et al. Identification of the spinocerebellar ataxia type 2 gene using a direct identification of repeat expansion and cloning technique, DIRECT. Nat Genet 1996;13:277-84.

9 Kawaguchi Y, Okamoto T, Taniwaki M, et al. CAG/CTG expansions in a novel gene for Machado-Joseph disease at expansions in a novel gene for Machado-Joseph
chromosome 13q32.1. Nat Genet 1994;8:221-7.

10 Zhuchenko O, Bailey J, Bonnen P, et al. Autosoma dominant cerebellar ataxia (SCA6) associated with small polyglutamine expansions in the $\alpha_{1 \mathrm{~A}}$-voltage-dependent calcium channel. Nat Genet 1997;15:62-9.

11 Koide R, Ikeuchi $\mathrm{T}$, Onodera $\mathrm{O}$, et al. Unstable expansion of CAG/CTG repeat in hereditary dentatorubralpallidoluysian atrophy (DRPLA). Nat Genet 1994;6:9-13.

12 Nagafuchi $S$, Yanagisawa $\mathrm{H}$, Sato $\mathrm{K}$, et al. Dentatorubra and pallidoluysian atrophy: expansion of an unstable CAG/ CTG trinucleotide on chromosome $12 \mathrm{p}$. Nat Genet 1994;6:13-18.

13 Matilla T, Volpini V, Genís D, et al. Presymptomatic analysis of spinocerebellar ataxia type 1 (SCA1) via the expansion of spinocerebellar ataxia type 1 (SCA1) via the expansion of the SCA1 CAG/CTG-repeat in a large pedigree displaying anticipation

14 Ikeuchi $\mathrm{T}$, Koide $\mathrm{R}$, Tanaka $\mathrm{H}$, et al. Dentatorubralpallidoluysian atrophy (DRPLA): clinical features are closely related to unstable expansions of trinucleotide (CAG/CTG) repeat. Ann Neurol 1995;37:769-75.

15 Maruyama H, Nakamura S, Matsuyama Z, et al. Molecular features of the CAG/CTG repeats and clinical manifestation of Machado-Joseph disease. Hum Mol Genet 1995;4: 807-12.

16 Benomar A, Krols L, Stevanin G, et al. The gene for autosomal dominant cerebellar ataxia with pigmentary macular dystrophy maps to chromosome 3p12-p21.1. Nat Genet 1995;10:84-8.

17 Gouw LG, Kaplan CD, Haines JH, et al. Retinal degeneration characterizes a spinocerebellar ataxia mapping to tion characterizes a spinocerebellar ataxi
chromosome 3p. Nat Genet 1995;10:89-93.
18 Ranum LPW, Schut LJ, Lundgren JK, Orr TH, Livingston DM. Spinocerebellar ataxia type 5 in a family descended DM. Spinocerebellar ataxia type 5 in a family descended from the grandparents of President Linc

19 Flanigan K, Gardner K, Alderson K, et al. Autosomal dominant spinocerebellar ataxia with sensory axonal neuropathy (SCA4): clinical description and genetic localization to chromosome 16q22.1. Am $\mathcal{F}$ Hum Genet 1996;59:392-9.

20 Trottier Y, Lutz Y, Stevanin G, et al. Polyglutamine expansion as a pathological epitope in Huntington's disease and four dominant cerebellar ataxias. Nature 1995;378: 403-6.

21 Lindblad K, Savontaus ML, Stevanin G, et al. An expanded CAG/CTG repeat sequence in spinocerebellar ataxia type 7. Genome Res 1996;6:965-71.

22 Schalling $M$, Hudson TJ, Buetow $\mathrm{KH}$, Housman DE. Direct detection of novel expanded trinucleotide repeats in the human genome. Nat Genet 1993;4:135-9.

23 Hofferbert S, Schanen NC, Chehab F, Francke U. Trinucleotide repeats in human genome: size distributions for all possible triplets and detection of expanded disease alleles in a group of Huntington disease individuals by the repeat expansion detection method. Hum Mol Genet 1997;6:7783.

24 Carero-Valenzuela R, Lindblad K, Payami H, et al. No evidence for association of familial Parkinson's disease with CAG/CTG repeat expansion. Neurology 1995;45:1760-3.

25 Morris AG, Gaitonde E, McKenna PJ, Mollon JD, Hunt DM. CAG/CTG repeat expansions and schizophrenia: association with disease in females and with early age-at-onset. Hum Mol Genet 1995;4:1957-61.

26 O'Donovan MC, Guy C, Craddock N, et al. Expanded CAG/CTG repeats in schizophrenia and bipolar disorder. Nat Genet 1995;10:380-1.

27 Petronis A, Bassett AS, Honer WG, et al. Search for unstable DNA in schizophrenia families with evidence for genetic anticipation. Am f Hum Genet 1996;59:905-11.

28 Bowen T, Guy C, Speight G, et al. Expansion of 50 CAG/CTG/CTG repeats excluded in schizophrenia by application of a highly efficient approach using repeat application of a highly efficient approach using repeat Genet 1996;59:912-17.

29 Martorell L, Pujana MA, Volpini V, et al. The repeat expansion detection (RED) method throughout the analysis of diseases with CAG/CTG/GTC repeat expansion: usefulness and limitations. Hum Mutat (in press)

30 Sirugo G, Kidd KK. Repeat expansion detection using Ampligase $^{\mathrm{X}}$ thermostable DNA ligase. Epicentre Forum 1995;2:1-3.

31 Stevanin G, Trottier Y, Cancel G, et al Screening for proteins with polyglutamine expansions in autosomal dominant with polyglutamine expansions in autosomal don
cerebellar ataxias. Hum Mol Genet 1996;5:1887-92. 